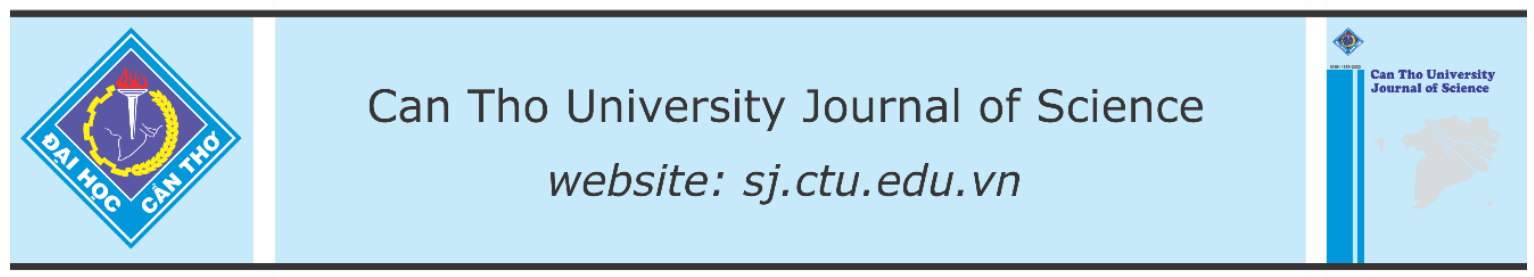

DOI: 10.22144/ctu.jen.2020.023

\title{
FACILE SYNTHESIS OF BIMETALLIC ZnCo-ZIFs AND Ag NANOPARTICLES LOADING ON ZnCo-ZIFs (Ag/ZnCo-ZIFs)
}

\author{
Dang Huynh Giao ${ }^{1 *}$, Le Thi Anh Thu ${ }^{1}$, Nguyen Huynh Thu Thao ${ }^{1}$, Ho Ngoc Tri Tan ${ }^{1}$, Doan Van \\ Hong Thien ${ }^{1}$ and Pham Van Toan ${ }^{2}$ \\ ${ }^{1}$ College of Engineering Technology, Can Tho University, Vietnam \\ ${ }^{2}$ College of Environment and Natural Resources, Can Tho University, Vietnam \\ *Correspondence: Dang Huynh Giao (email: dhgiao@ctu.edu.vn)
}

\section{Article info.}

Received 21 May 2020

Revised 21 Jun 2020

Accepted 30 Nov 2020

\section{Keywords}

Ag/ZnCo-ZIFs, bimetallic, nanoparticles, zeolitic imidazole frameworks, ZnCo-ZIFs

\begin{abstract}
A kind of bimetallic Zn/Co zeolitic imidazole frameworks (ZnCo-ZIFs) was successfully synthesized from 2-methylimidazole and two metal salts including zinc nitrate, cobalt (II) nitrate by a solvothermal method at room temperature. Subsequently, Ag nanoparticles (AgNPs) loading on bimetallic frameworks were prepared by a facile impregnation method in acetone solvent. Both ZnCo-ZIFs and Ag/ZnCo-ZIFs were analyzed by several characterization techniques including powder X-ray diffraction (PXRD), scanning electron microscope (SEM), Fourier transform infrared spectroscopy $(F T-I R)$, thermogravimetric analysis $(T G A)$ and energy-dispersive $X$ ray spectroscopy (EDX). The results showed that ZnCo-ZIFs crystals had sodalite structure, high thermal stability, and AgNPs were successfully loaded on the ZnCo-ZIFs with content of $18.42 \%$. Ag/ZnCo-ZIFs in this combination may pave a way for preparing a kind of heterogeneous catalyst to remove organic compounds from aqueous solutions.
\end{abstract}

Cited as: Giao, D.H., Thu, L.T.A., Thao, N.H.T., Tan, H.N.T., Thien, D.V.H. and Toan, P.V., 2020. Facile synthesis of bimetallic ZnCo-ZIFs and Ag nanoparticles loading on ZnCo-ZIFs (Ag/ZnCo-ZIFs). Can Tho University Journal of Science. 12(3): 47-53.

\section{INTRODUCTION}

Zeolitic imidazole frameworks (ZIFs) are a subclass of metal organic frameworks (MOFs) that were formed from both inorganic and organic components, namely transition metal ions $\left(\mathrm{M}^{2+}=\mathrm{Zn}^{2+}\right.$ or $\left.\mathrm{Co}^{2+}\right)$ and organic imidazolate linkers ( $\mathrm{Im}=$ Imidazole) (Gross et al., 2012). The unique properties of ZIFs such as high surface areas, excellent thermal and chemical stability help them to have been widely used for several potential applications including gas storage (Assfour et al., 2011) and separation (Liu and Smit, 2010), adsorption (Du et al.,
2017; Zhang et al., 2019) as well as catalysis (Nagarjun and Dhakshinamoorthy, 2019). One of the recently interested ZIFs compounds is $\mathrm{ZnCo}$-ZIFs which are composed of $\mathrm{Zn}^{2+}, \mathrm{Co}^{2+}$ cations and 2-methylimidazole anions with a sodalite-related structure (Han et al., 2019). Notably, syntheses of bimetallic ZnCo-ZIFs frameworks have more outstanding characteristics than monometallic (e.g., ZIF-8, ZIF-67) such as the increase in pore volume and surface area (Kaur et al., 2016).

In science and engineering, nanotechnology is rapidly developed because of their emergent physio- 
chemical properties. The intrinsic features of nanoparticles (NPs) depend on the size, shape, surface functional groups and crystalline structure of them (Vreeland et al., 2015). However, NPs have a high surface energy which makes them easy to agglomerate and form larger blocks (Guo and Li, 2004; Lee et al., 2008). That is one of the reasons why the combination of NPs and ZIFs has been more and more attractive to scientists in the world. ZIFs with porous structure can encapsulate NPs (designated NPs@ZIFs) or NPs can attach on the large surface area of ZIFs (designated NPs/ZIFs) and the aggregation can be remarkably relieved, thus preserving the chemical activity and stability of the NPs (Jiang et al., 2011). Moreover, NPs combined with ZIFs can create a new material that integrates both the advantages of each individual component and cannot be obtained from single-component material. In recent years, the materials combined of NPs and ZIFs have considered with different synthesis methods as well as different applications, typically about catalysis (Dhakshinamoorthy and Garcia, 2012). For instance, in 2014, Huang and co-workers demonstrated a facile synthetic strategy to prepare bimetallic alloy nanocrystals were encapsulated in ZIF-8, namely PtPd@ZIF-8 and used for synergistic catalysis of ethylene oxidative degradation. After that, $\mathrm{Li}$ et al. (2016) reported to synthesize Pd@ZIF-67 to be used as heterogeneous catalyst for $\mathrm{Cr}$ (VI) reduction to $\mathrm{Cr}$ (III). The PdNPs could be encapsulated into the uniformly shaped ZIF-67 through the reduction of HCOOH. The as-synthesized Pd@ZIF-67 material had a high catalytic activity and excellent cycle durability which could be reused for 10 times.

Among all the nanomaterials, silver has got more attention because of their optical, electronic and magnetic properties. Silver nanoparticles (AgNPs) seem to be found the most applications in the industry ( $\mathrm{Li}$ et al., 2012). Indeed, AgNPs have many applications such as antibacterial (El-Rafie et al., 2010), conductivity (Hebeish et al., 2015), optical sensor (Kang et al., 2014), especially catalytic (Joseph and Mathew, 2015). Therefore, herein, a simple and effective method was reported for preparing of $\mathrm{ZnCo}$ ZIFs and $\mathrm{Ag} / \mathrm{ZnCo}-\mathrm{ZIFs}$ which are expected to be a potential heterogeneous catalyst.

\section{MATERIALS AND METHODS}

\subsection{Materials}

All reagents and starting materials were purchased from Sigma-Aldrich and Acros, and used as received without further purification.

\subsection{Preparation of $\mathrm{ZnCo-ZIFs}$}

ZnCo-ZIFs were synthesized at ambient temperature in methanol solvent. The ratio of cobalt nitrate and zinc nitrate was fixed to $3: 1$ as previously reported (Kaur et al., 2016). Typically, $\mathrm{Co}\left(\mathrm{NO}_{3}\right)_{2} \cdot 6 \mathrm{H}_{2} \mathrm{O}(0.873 \mathrm{~g}, 3 \mathrm{mmol}), \mathrm{Zn}\left(\mathrm{NO}_{3}\right)_{2} \cdot 6 \mathrm{H}_{2} \mathrm{O}$ $(0.297 \mathrm{~g}, 1 \mathrm{mmol})$ and 2-MIm (2-MIm=2-methylimidazole; $1.3136 \mathrm{~g}, 16 \mathrm{mmol}$ ) were respectively dissolved in methanol $(10 \mathrm{~mL})$. Then, zinc nitrate was slowly added to the cobalt nitrate and magnetic stirring during $15 \mathrm{~min}$ to form a homogeneous mixture. Next, this mixture was dropped in 2-MIm solution, resulting in the formation of a purple suspension and they were maintained at room temperature for $24 \mathrm{~h}$. After that, the purple precipitation was obtained by centrifugation, washed with $\mathrm{MeOH}(3 \times 10 \mathrm{~mL})$ for 3 days and dried at $60^{\circ} \mathrm{C}$. The molar of two metal salts and ligand was altered by varying the initial concentration of mixture salts and 2-MIm $(\mathrm{Zn} / \mathrm{Co}: 2-$ MIm molar ratio $=1: 2,1: 4,1: 6,1: 8,1: 10)$.

\subsection{Preparation of $\mathrm{Ag} / \mathrm{ZnCo}-\mathrm{ZIFs}$}

Before loading Ag nanoparticles, ZnCo-ZIFs was heated at $56^{\circ} \mathrm{C}$ for $5 \mathrm{~h}$ to obtain optimally evacuated sample. Then, $\mathrm{Ag} / \mathrm{ZnCo}-\mathrm{ZIFs}$ catalyst was prepared by a wet impregnation method with $\mathrm{AgNO}_{3}$ as the metal salt and acetone as the impregnation solvent. The mass ratio of $\mathrm{AgNO}_{3}$ and $\mathrm{ZnCo}$-ZIFs was fixed to $1: 4$. Briefly, $62.5 \mathrm{mg}$ of silver nitrate $\left(\mathrm{AgNO}_{3}\right)$ was dissolved in $30 \mathrm{~mL}$ of acetone, then $250 \mathrm{mg}$ of $\mathrm{ZnCo-ZIFs} \mathrm{crystals} \mathrm{were} \mathrm{added} \mathrm{into} \mathrm{the} \mathrm{solution} \mathrm{un-}$ der magnetic stirring for $1 \mathrm{~h}$ at $56^{\circ} \mathrm{C}$. Subsequently, $0.25 \mathrm{~mL}$ of formic acid $(\mathrm{HCOOH})$ was slowly added into the mixture with magnetic stirring, resulting in the dark solid formed. The sample after synthesizing was separated through centrifugation, washing with acetone several times and drying at $60^{\circ} \mathrm{C}$ for $8 \mathrm{~h}$ to obtain the $\mathrm{Ag} / \mathrm{ZnCo}$-ZIFs.

\section{RESULTS AND DISCUSSION}

\subsection{Characterization of the $\mathrm{ZnCo}-\mathrm{ZIFs}$ and Ag/ZnCo-ZIFs}

Powder X-ray diffraction (PXRD) patterns of the synthesized ZnCo-ZIFs displayed sharp and prominent peaks analogous to simulation, ZIF-8 and ZIF67 which confirmed the phase purity of the synthesized. Although five samples were synthesized in different ratios, PXRD results are almost the same (Fig. 1). The clearly peaks indexed to $2 \theta$ values of $7.3^{\circ}, 10.4^{\circ}, 12.7^{\circ}, 14.8^{\circ}, 16.5^{\circ}, 18^{\circ}, 22.1^{\circ}, 24.5^{\circ}$, $25.5^{\circ}, 26.7^{\circ}$ and $29.6^{\circ}$ corresponding to (011), (002), (112), (022), (013), (222), (114), (233), (224), (134) 
and (044), respectively having a good match with previously reported results (Zhou et al., 2017).

Besides, when increasing the ratio of metal salts and 2-MIm in the synthesis, the process yield increased (Fig. 2). The yield of ZnCo-ZIFs-1:8 was about $52 \%$, dramatically increased against the ZnCo-ZIFs1:2 (10\%), ZnCo-ZIFs-1:4 (29\%), ZnCo-ZIFs-1:6
(30\%), respectively and only lower ZnCo-ZIFs-1:10 was $8 \%$ (the yield of ZnCo-ZIFs-1:10 achieved $60 \%$ ). This proved directly affection of ligand to the formation of bimetallic crystals due to its linking role in the structure of ZIFs. Economically, the ratio of metal salts and ligand selected for the optimal synthesis condition is $1: 8$.

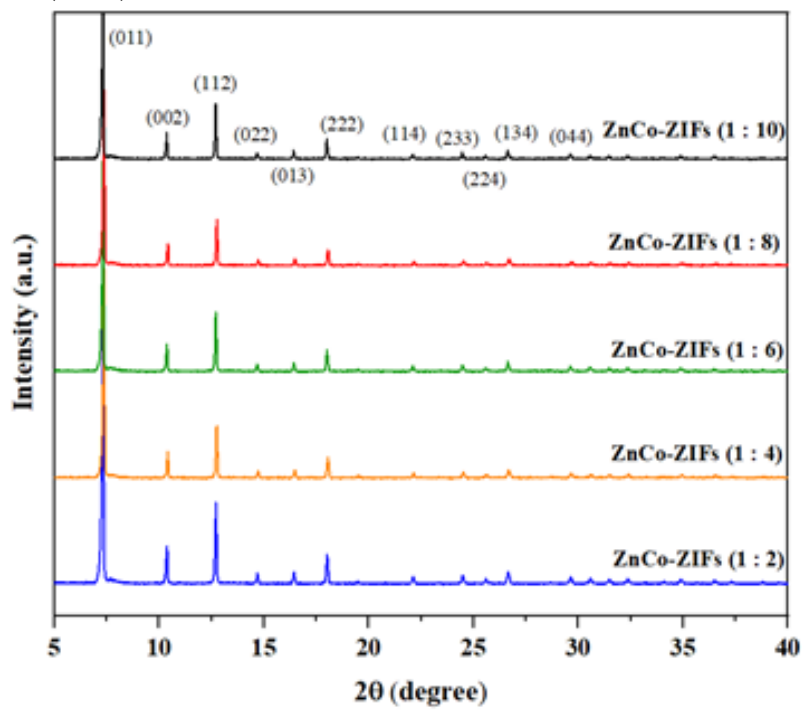

Fig. 1: PXRD patterns of bimetallic ZnCo-ZIFs samples with different molar ratios of mixture metal salts and 2-MIm

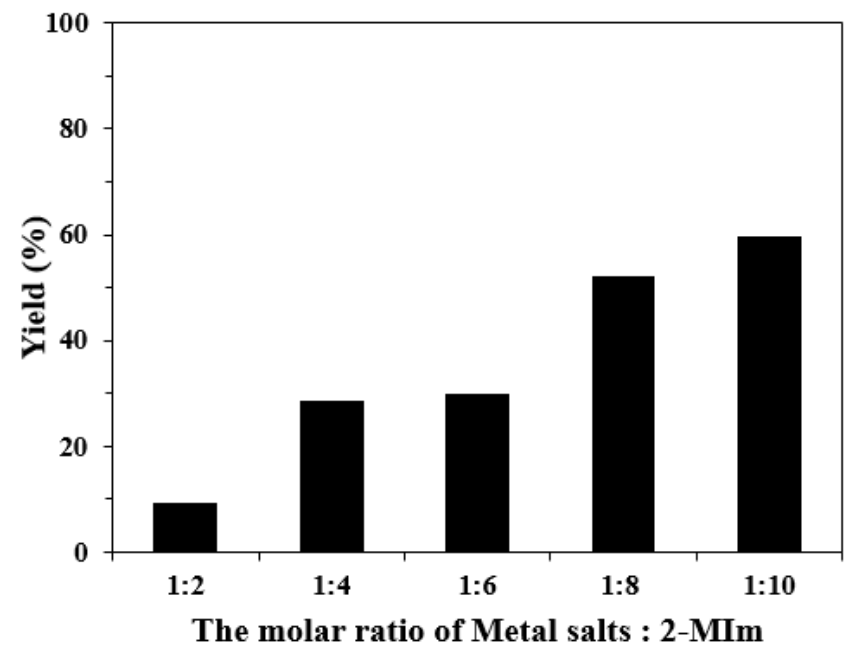

Fig. 2: The yield of ZnCo-ZIFs with different molar ratios of mixture metal salts and 2-MIm

Narrow and strong peaks are obtained from the assynthesized ZnCo-ZIFs and Ag/ZnCo-ZIFs showed their high crystallinity (Fig. 3). Furthermore, $\mathrm{Ag} / \mathrm{ZnCo}$-ZIFs sample appeared the sharp and clear peaks at $2 \theta$ values of $38.1^{\circ}, 44.3^{\circ}, 64.5^{\circ}, 77.4^{\circ}$ corresponding to (111), (200), (220) and (311), respectively (Fig. 3a). This is entirely consistent with previously reported for the presence of AgNPs (Park et al., 2011). With the participation of silver, PXRD of Ag/ZnCo-ZIFs sample displayed that the structure of $\mathrm{ZnCo-ZIFs}$ was only partially maintained with weak intensity. To clarify about presence of silver, EDX and SEM were defined. 


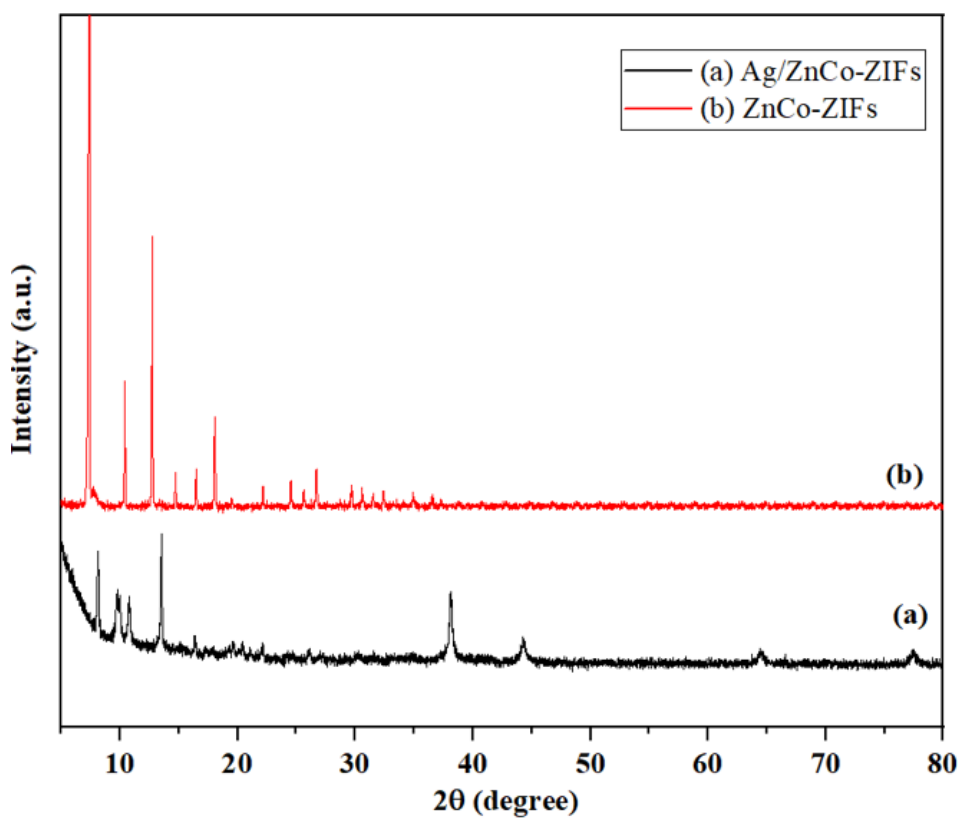

Fig. 3: PXRD of (a) Ag/ZnCo-ZIFs, (b) ZnCo-ZIFs-1:8

EDX analysis was conducted to determine the elemental composition of the nanoparticles. The EDX of $\mathrm{ZnCo}$-ZIFs demonstrated the presence and distribution of $\mathrm{C}, \mathrm{N}, \mathrm{Co}$ and $\mathrm{Zn}$

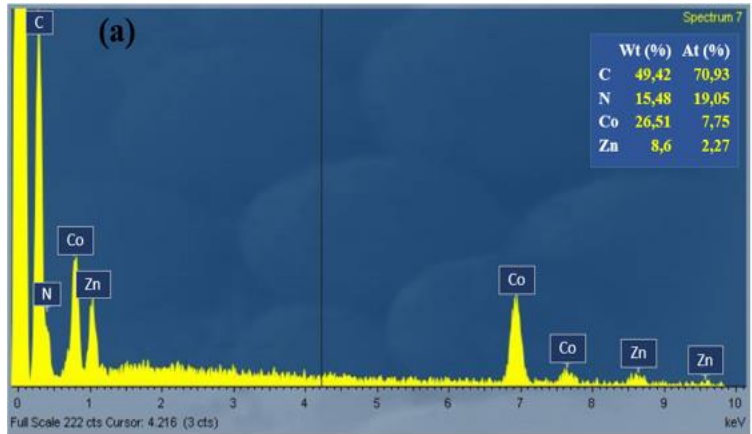

(Fig. 4a). Besides the elements in the original bimetallic frameworks, EDX of $\mathrm{Ag} / \mathrm{ZnCo}-\mathrm{ZIFs}$ had the appearance of $\mathrm{Ag}$, this also demonstrated the successful reduction of formic acid to silver nitrate (Fig. $4 b)$.

Fig. 4: EDX spectra of (a) ZnCo-ZIFs, (b) Ag/ZnCo-ZIFs

Fig. 5 showed the SEM micrographs of representative $\mathrm{Ag} / \mathrm{ZnCo}$-ZIFs sample at the different magnification ratios (a), (b), (c) and ZnCo-ZIFs (d), respectively. The results revealed that ZnCo-ZIFs had a

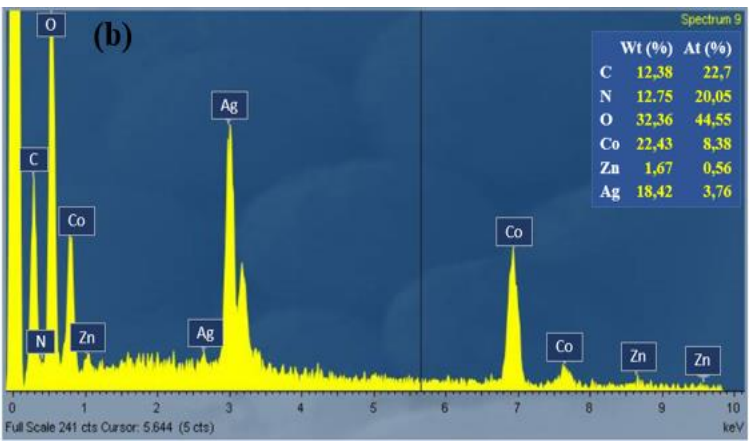

polyhedron structure, meanwhile on the surface $\mathrm{Ag} / \mathrm{ZnCo}-\mathrm{ZIFs}$ had appearance of tiny particles that predicted to be silver particles. 

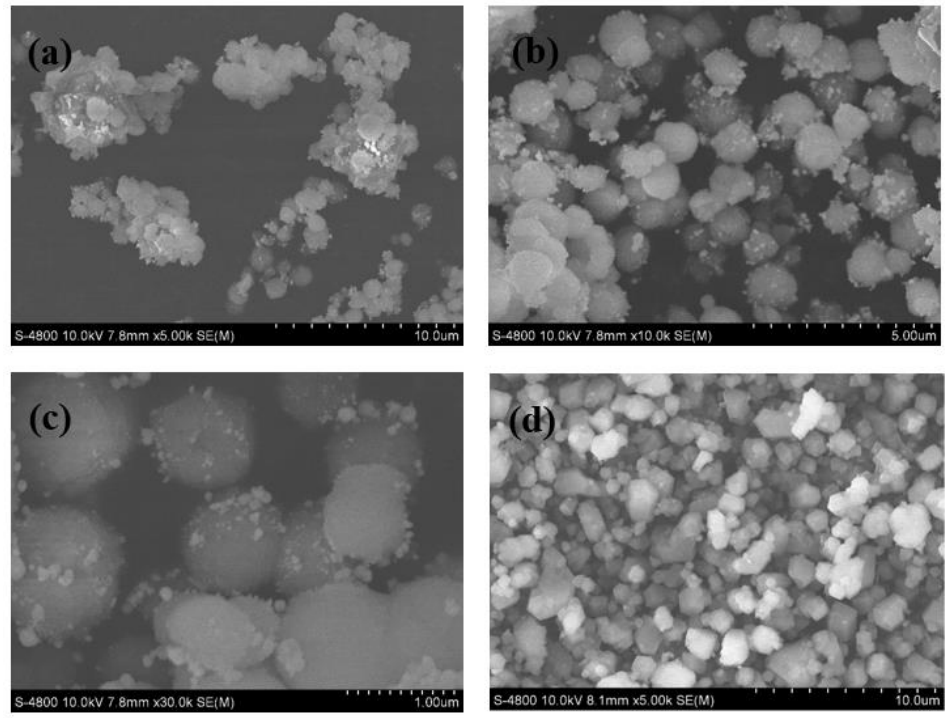

Fig. 5: SEM micrographs of Ag/ZnCo-ZIFs at the different magnification ratios (a) x5000, (b) x10000, (c) $\mathrm{x30000}$, (d) ZnCo-ZIFs

For more detailed investigation about properties of materials, Fig. 6 illustrated FT-IR spectra of the molecular structure of $\mathrm{Ag} / \mathrm{ZnCo}-\mathrm{ZIFs}$, compared to ZnCo-ZIFs and 2-MIm. The FT-IR spectra of linker 2-MIm showed the major absorbance peak was observed at $3400-2200 \mathrm{~cm}^{-1}$ representing the $\mathrm{N}-\mathrm{H}$ stretching vibration at $1846 \mathrm{~cm}^{-1}$ (Hachuła et al., 2010) while it completely disappeared in the FTIR of $\mathrm{ZnCo}-\mathrm{ZIFs}$ and $\mathrm{Ag} / \mathrm{ZnCo}-\mathrm{ZIFs}$, indicating that $\mathrm{N}-\mathrm{H}$ bond of 2-MIm were severed to upon coordination with metal ions. On the spectrum of $\mathrm{Ag} / \mathrm{ZnCo}$-ZIFs, peaks appeared in the range of 600$1700 \mathrm{~cm}^{-1}$ which could be indicated to the stretching and bending frequency of the imidazole ring. These peaks almost corresponded to the peaks of 2-MIm as well as ZnCo-ZIFs, they had a little difference but it was insignificant. Indeed, the $\mathrm{C}=\mathrm{N}$ bond of 2-MIm appeared at $1594 \mathrm{~cm}^{-1}$ while on ZnCo-ZIFs, $\mathrm{Ag} / \mathrm{ZnCo}$-ZIFs corresponding $1579 \mathrm{~cm}^{-1}, 1589 \mathrm{~cm}^{-}$ 1 , respectively. The difference between $\mathrm{Ag} / \mathrm{ZnCo}-$ ZIFs and ZnCo-ZIFs was only about $0.63 \%$, similar with other links. The decrease in peak intensity might be due to the presence of $\mathrm{Ag}$ that covers the surface of $\mathrm{ZnCo}$-ZIFs, which had an impact on energy absorption and emission, reducing the signal strength as well as the movement of the peak.

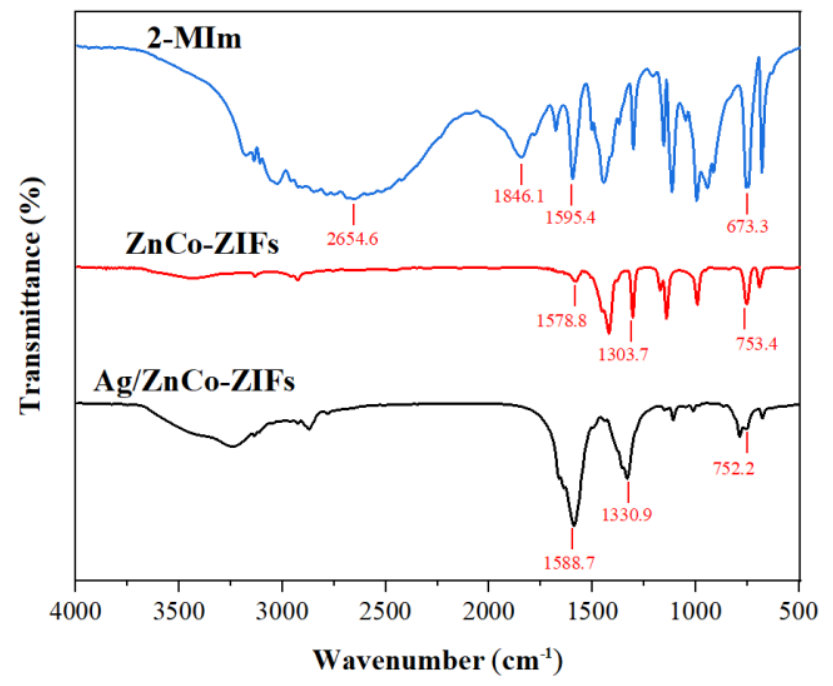

Fig. 6: FT-IR spectra of 2-MIm, ZnCo-ZIFs, Ag/ZnCo-ZIFs 
Furthermore, TGA measurements of ZnCo-ZIFs and $\mathrm{Ag} / \mathrm{ZnCo}$-ZIFs were performed from $30^{\circ} \mathrm{C}$ to $800^{\circ} \mathrm{C}$ (Fig. 7). For ZnCo-ZIFs, in the published literature, initial weight loss of $2-6 \%$ was observed from $135-155^{\circ} \mathrm{C}$, from the removal of residual solvent or unreacted 2-MIm (Kaur et al., 2016). In this study, the mass loss occurred mainly at temperatures from $400-720^{\circ} \mathrm{C}$, resulting from decomposition of organic linkers. This showed that ZnCo-ZIFs had a stable structure over a wide temperature range. Thermal resistance of $\mathrm{ZnCo-ZIFs} \mathrm{is} \mathrm{considered} \mathrm{to}$ be superior to ZIF-67 and ZIF-8 (Kaur et al., 2016). For Ag/ZnCo-ZIFs, initial weight loss of $2.756 \%$ at temperature from $30-115^{\circ} \mathrm{C}$, this might come from the loss of small molecules or residual acetone solvent. The next weight loss observed from 115$300^{\circ} \mathrm{C}, \quad 300-360^{\circ} \mathrm{C}$ corresponding to $35.96 \%$, $5.185 \%$, respectively. $\mathrm{Up}$ to $460^{\circ} \mathrm{C}, \mathrm{Ag} / \mathrm{ZnCo}-\mathrm{ZIFs}$ almost completely decomposed and remaining mass were only about $40.18 \%$. Generally, the TGA trace for $\mathrm{ZnCo}$-ZIFs revealed a high decomposition temperature of $530^{\circ} \mathrm{C}$ while $\mathrm{Ag} / \mathrm{ZIF}-67$ were approximately $220^{\circ} \mathrm{C}$. This could be explained that in the synthesis process, $\mathrm{ZnCo-ZIFs} \mathrm{took} \mathrm{an} \mathrm{hour} \mathrm{to} \mathrm{dis-}$ perse in acetone at $60^{\circ} \mathrm{C}$, moreover $\mathrm{Ag}^{+}$ions were reduced to $\mathrm{Ag}^{0}$ by $\mathrm{HCOOH}$. The contact of material with reducing agent under heating stirring conditions, reaction time of more than 90 min might make them lose stability.

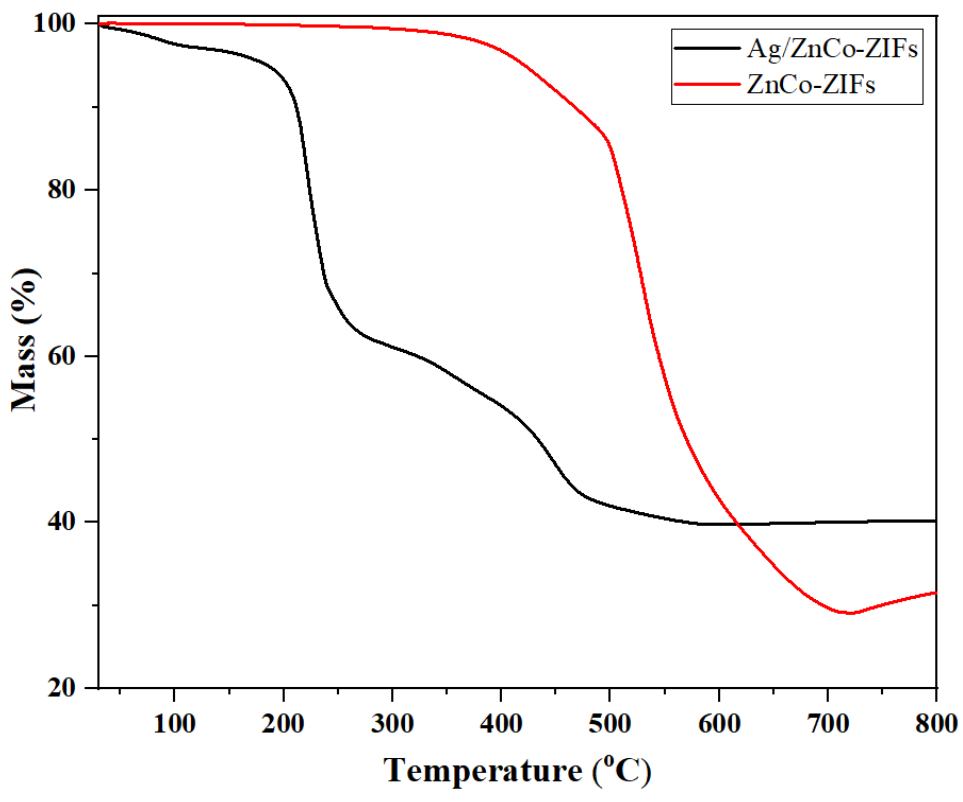

Fig. 7: TGA curves of ZnCo-ZIFs and Ag/ZnCo-ZIFs

\section{CONCLUSIONS}

In summary, bimetallic $\mathrm{Zn} / \mathrm{Co}$ zeolitic imidazole frameworks (ZnCo-ZIFs) were successfully synthesized by facile solvothermal method at ambient temperature with the yield over $52 \%$ being obtained. $\mathrm{Bi}-$ metallic ZnCo-ZIFs were determined to be have similar structure as monometallic ZIF-67 and ZIF8. Furthermore, this solid was stable in wide temperature range. Besides, Ag NPs were successfully loaded on the surface of ZnCo-ZIFs by using an impregnation-reduction procedure and form to the $\mathrm{Ag} / \mathrm{ZnCo}-\mathrm{ZIFs}$ material. With the unique and individual properties of silver and bimetallic framework as well as this successful combination, $\mathrm{Ag} / \mathrm{ZnCo}$ -
ZIFs are expected to be potential heterogeneous catalyst in removing the persistent organic compounds in the water.

\section{ACKNOWLEDGMENTS}

This study is funded in part by the Can Tho University Improvement Project VN14-P6, supported by a Japanese ODA loan.

\section{REFERENCES}

Assfour, B., Leoni, S., Yurchenko, S., and Seifert, G., 2011. Hydrogen storage in zeolite imidazolate frameworks. A multiscale theoretical investigation. International Journal of Hydrogen Energy. 36(10): 6005-6013.

Dhakshinamoorthy, A., and Garcia, H., 2012. Catalysis by metal nanoparticles embedded on metal-organic 
frameworks. Chemical Society Reviews. 41(15): 5262-5284.

Du, X. D., Wang, C. C., Liu, J. G., et al., 2017. Extensive and selective adsorption of ZIF-67 towards organic dyes: performance and mechanism. Journal of Colloid and Interface Science. 506: 437-441.

El-Rafie, M. H., Mohamed, A. A., Shaheen, T. I., and Hebeish, A., 2010. Antimicrobial effect of silver nanoparticles produced by fungal process on cotton fabrics. Carbohydrate Polymers. 80(3): 779-782.

Gross, A. F., Sherman, E., and Vajo, J. J., 2012. Aqueous room temperature synthesis of cobalt and zinc sodalite zeolitic imidazolate frameworks. Dalton Transactions. 41(18): 5458-5460.

Guo, D. J., and Li, H. L., 2004. Electrochemical synthesis of Pd nanoparticles on functional MWNT surfaces. Electrochemistry Communications. 6(10): 999-1003.

Hachuła, B., Nowak, M., and Kusz, J., 2010. Crystal and molecular structure analysis of 2-methylimidazole. Journal of Chemical Crystallography. 40(3): 201-206.

Han, X., Ling, X., Wang, Y., et al., 2019. Generation of nanoparticle, atomic-cluster, and single-atom cobalt catalysts from zeolitic imidazole frameworks by spatial isolation and their use in zinc-air batteries. Angewandte Chemie. 131(16): 5413-5418.

Hebeish, A., Farag, S., Sharaf, S., and Shaheen, T. I., 2015. Nanosized carbamoylethylated cellulose as novel precursor for preparation of metal nanoparticles. Fibers and Polymers. 16(2): 276-284.

Huang, Y., Zhang, Y., Chen, X., Wu, D., Yi, Z., and Cao, R., 2014. Bimetallic alloy nanocrystals encapsulated in ZIF-8 for synergistic catalysis of ethylene oxidative degradation. Chemical Communications. 50(70): 10115-10117.

Jiang, H. L., Akita, T., Ishida, T., Haruta, M., and Xu, Q., 2011. Synergistic catalysis of Au@ Ag core-shell nanoparticles stabilized on metal-organic framework. Journal of the American Chemical Society. 133(5): 1304-1306.

Joseph, S., and Mathew, B., 2015. Microwave-assisted green synthesis of silver nanoparticles and the study on catalytic activity in the degradation of dyes. Journal of Molecular Liquids. 204: 184-191.

Kang, Y., Wu, T., Liu, B., Wang, X., and Du, Y., 2014. Selective determination of mercury (II) by self-referenced surface-enhanced Raman scattering using dialkyne-modified silver nanoparticles. Microchimica Acta. 181(11-12): 1333-1339.
Kaur, G., Rai, R. K., Tyagi, D., et al., 2016. Room-temperature synthesis of bimetallic $\mathrm{Co}-\mathrm{Zn}$ based zeolitic imidazolate frameworks in water for enhanced $\mathrm{CO}_{2}$ and $\mathrm{H}_{2}$ uptakes. Journal of Materials Chemistry A. 4(39): 14932-14938.

Lee, Y., Choi, J. R., Lee, K. J., Stott, N. E., and Kim, D, 2008. Large-scale synthesis of copper nanoparticles by chemically controlled reduction for applications of inkjet-printed electronics. Nanotechnology. 19(41): 415604.

Li, H. C., Liu, W. J., Han, H. X., and Yu, H. Q., 2016. Hydrophilic swellable metal-organic framework encapsulated Pd nanoparticles as an efficient catalyst for $\mathrm{Cr}$ (VI) reduction. Journal of Materials Chemistry A. 4(30): 11680-11687.

Li, H. J., Zhang, A. Q., Hu, Y., Sui, L., Qian, D. J., and Chen, M., 2012. Large-scale synthesis and self-organization of silver nanoparticles with Tween 80 as a reductant and stabilizer. Nanoscale Research Letters. 7(1): 1-13.

Liu, B., and Smit, B., 2010. Molecular simulation studies of separation of $\mathrm{CO}_{2} / \mathrm{N}_{2}, \mathrm{CO}_{2} / \mathrm{CH}_{4}$, and $\mathrm{CH}_{4} / \mathrm{N}_{2}$ by ZIFs. The Journal of Physical Chemistry C. 114(18): 8515-8522.

Nagarjun, N., and Dhakshinamoorthy, A., 2019. A CuDoped ZIF-8 metal organic framework as a heterogeneous solid catalyst for aerobic oxidation of benzylic hydrocarbons. New Journal of Chemistry. 43(47): 18702-18712.

Park, E. J., Lee, S. W., Bang, I. C., and Park, H. W., 2011. Optimal synthesis and characterization of Ag nanofluids by electrical explosion of wires in liquids. Nanoscale Research Letters. 6(1): 223.

Vreeland, E. C., Watt, J., Schober, G. B., et al., 2015. Enhanced nanoparticle size control by extending LaMer's mechanism. Chemistry of Materials. 27(17): 6059-6066.

Zhang, J., Hu, X., Yan, X., Feng, R., Zhou, M., and Xue, J., 2019. Enhanced adsorption of Rhodamine B by magnetic nitrogen-doped porous carbon prepared from bimetallic ZIFs. Colloids and Surfaces A: Physicochemical and Engineering Aspects. 575: 10-17.

Zhou, K., Mousavi, B., Luo, Z., Phatanasri, S., Chaemchuen, S., and Verpoort, F., 2017. Characterization and properties of $\mathrm{Zn} / \mathrm{Co}$ zeolitic imidazolate frameworks vs. ZIF-8 and ZIF-67. Journal of Materials Chemistry A. 5(3): 952-957. 